\title{
Vernetzung der Hydrogeologie mit Nachbardisziplinen: eine Zukunftsoption?
}

\author{
Thomas Himmelsbach \\ Eingang des Beitrages: 27. 12.2008/Online veröffentlicht: 6.2.2009 \\ (C) Springer-Verlag 2009
}

Liebe Leserinnen und Leser!

Auf der IAH-Tagung 2008 in Toyama, Japan, wurde unser ehemaliger FH-DGG-Vorsitzender Willi Struckmeier zum neuen IAH-Präsidenten gewählt. Die IAH ist mit derzeit 3.500 Mitgliedern aus 135 Ländern die wohl bedeutendste internationale Institution auf dem Grundwassersektor. Aufgrund seiner langjährigen Mitgliedschaft sowie seinen vielfältigen Aufgaben innerhalb der IAH, zunächst als Vice President Western Europe und anschließend Secretary General, ist Willi Struckmeier der beste Kandidat, um die IAH weiter $\mathrm{zu}$ entwickeln und im internationalen Konzert der auf dem Wassersektor tätigen Organisationen zu verankern. Stellvertretend für die deutschen Mitglieder der IAH und im Namen der Mitglieder der FH-DGG möchte ich ihm ganz herzlich zur Wahl zum IAH-Präsidenten gratulieren und alles Gute für dieses sicher nicht einfache Amt wünschen. Ich habe den Einstieg in mein Editorial über die IAH bewusst gewählt. Zum einen war es mir ein persönliches Anliegen die Glückwünsche auszusprechen, zum anderen sind wir so bereits in die Thematik meines Editorials eingetaucht. Der erste gemeinsame Fachkongress von IAHS und IAH im September 2009 in Hyderabad, Indien, stellt ein Beispiel für die zunehmende

PD Dr. Thomas Himmelsbach $(\square)$ Bundesanstalt für Geowissenschaften und Rohstoffe Hannover,

Fachbereich Grundwasserressourcen Stilleweg 2, 30655 Hannover, Deutschland
Bedeutung der internationalen Vernetzung der Hydrogeologie mit ihren Nachbardisziplinen dar. Wenn Sie das Programm der Tagung in Hyderabad (http://www.appliedhydrology.org/ iahs/iahshome.view) studieren, werden Sie feststellen, dass neben originären Themen der beteiligten Organisationen, neue Themen aufgegriffen werden, die zu einem großen Teil in der Diskussion über die Auswirkungen der weltweiten Klimaänderung verankert sind. Mario Schirmer hatte in seinem Editorial (Grundwasser 3/2008) bereits auf den „IPCC (Intergovernmental Panel on Climate Change) Climate Change 2007: Synthesis Report" hingewiesen. Ich möchte einige Aspekte aufgreifen um die Bedeutung der Vernetzung der Hydrogeologie mit ihren Nachbardisziplinen im Hinblick auf eine zielgerichtete Abminderung von weltweiten Klimafolgen herauszustellen.

Im Sommer 2008 fand in der ugandischen Hauptstadt Kampala die erste Internationale Konferenz „Groundwater \& Climate in Africa" statt. Die auf der Tagung vorgestellten, teils unterschiedlichen Klimaszenarien haben eines gemeinsam: sie verdeutlichen, dass es in unterschiedlichen Teilen von Afrika zu grundlegend verschiedenen Auswirkungen des Klimawandels kommen wird. Neben „Gewinnern“ der Klimaänderung, Länder die auf generell feuchtere Bedingungen hoffen können, wird es auch „Verlierer" geben, die sich auf trockenere Klimabedingungen einstellen müssen. Auch wenn die Änderungen in den jährlichen Nieder- schlagshöhen auf den ersten Blick marginal erscheinen, so haben sie dennoch immer großräumige Veränderungen der Vegetationszonen zur Folge. Neben der Änderung der absoluten Niederschlagsmengen kommt es auch zu einer zunehmenden Variabilität des Klimas. In vielen Entwicklungsländern bedeutet eine abnehmende Verlässlichkeit des Klimas eine große Herausforderung für niederschlagsbasierte Agrarwirtschaften, was sich auf die Nahrungsmittelsicherheit negativ auswirken kann. Infolge längerer Dürrephasen mit zeitweise auftretenden Starkniederschlagsperioden wird es in diesen Ländern daher fast zwangsläufig zu einer Ausweitung des Bewässerungslandbaues und somit zu einer verstärkten Grundwassernutzung kommen, da Oberflächengewässer nur während weniger Monate im Jahr zur Verfügung stehen. In Europa wird es infolge der steigenden Nachfrage nach Lebensmitteln, aber auch Bio-Treibstoffen, mittelfristig zu einer Steigerung der landwirtschaftlichen Produktion kommen. Diese Steigerung kann zum einen durch die Umwidmung ehemaliger Stilllegungsflächen erreicht werden, auf der anderen Seite wird es auch in Europa zu einer Ausweitung des Beregnungsfeldbaus kommen. So gibt es in den zentralen und südlichen Departments Frankreichs kaum noch ein Feld ohne Anschluss an großräumige Bewässerungsstrukturen (siehe: http:// www.cemagref.fr). Betrachtet man in diesem Licht die Bedingungen unserer östlichen Bundesländer, so entsteht hier für die Hydrogeologie ein neues 
Themenfeld, welches frühzeitig in enger Abstimmung mit Agraringenieuren, Hydrologen aber auch Vertretern der verschiedenen Europäischen Institutionen besetzt werden sollte. Dass dieses Themenfeld auf Europäischer Ebene bereits eine gestiegene Bedeutung hat, wird beim Studium des siebten Aufrufs zum Europäischen Forschungsprogramm deutlich.

Bei der Suche nach Grundwasser in Entwicklungsländern stehen Hydrogeologen oftmals vor einem unlösbaren Problem. In den meisten Fällen sind große Areale nur unzureichend durch Bohrungen erschlossen, und wenn es Bohrungen gibt, ist die Dokumentation über die erbohrten Schichten und die darin enthaltenen Grundwasserleiter lückenhaft. Im besten Fall wurde ein Pumpversuch durchgeführt, der aber nicht immer verlässliche Aussagen über die wahren hydraulischen Verhältnisse und die Ergiebigkeit des Grundwasserleiters liefert. Das Hauptproblem liegt jedoch darin, dass über die räumliche Verteilung der Grundwasserleiter zunächst nur wenige Informationen verfügbar sind und auch kein Geld für Bohrungen zur Verfügung steht. Erfreulicherweise hat die hydro-geophysikalische Erkundung mithilfe der klassischen geoelektrischen Verfahren, und neuerdings auch mit der Transienten-Elektromagnetik (TEM) und Magneto-Tellurik, große Fortschritte gemacht. Es stehen neue geophysikalische Verfahren bereit, die auch die Erkundung tief liegender Strukturen gestatten. Naturgemäß hat jedes der oben genannten Verfahren ganz individuelle Vor- und Nachteile, wobei sich deren Vorteile jedoch bei geschickter Kombination und Einbindung in die hydrogeologische Modellbildung gegenseitig ergänzen können. In der Anwendung können sich hieraus sehr spannende, neue Projekte ergeben. So wurden im Rahmen der technischen Zusammenarbeit in Namibia durch boden- und aerogeophysikalische Erkundungen Süßwasservor- kommen entdeckt, die unterhalb von versalzten Grundwasserleitern liegen und deren Existenz zunächst niemand für möglich gehalten hatte.

Ein weiteres Themenfeld, dem sich Hydrogeologen vor allem in Hinblick auf den Grundwasserschutz widmen sollten, ist die weltweite sanitäre Versorgungskrise. Weltweit leben ca. 2,5 Milliarden Menschen unter unwürdigen sanitären Bedingungen. Häufig verfügen sie über kein sauberes Trinkwasser, es mangelt an sanitären Einrichtungen und einem gezielten Wassermanagement. Da der Anschluss an die öffentliche Wasserversorgung aus finanziellen Gründen nicht erschwinglich oder aus technischen Gründen nicht möglich ist, sind insbesondere ärmere Bevölkerungsschichten in den schnell wachsenden Ballungsräumen zur Deckung ihres Trink- und Brauchwasserbedarfs auf einfache Handbrunnen angewiesen. Da das Grundwasser jedoch flächenhaft durch Fäkalkeime verunreinigt ist, treten verstärkt Durchfallerkrankungen auf, die vor allem für Kinder oft tödlich enden. So sterben jedes Jahr allein 2,2 Millionen Menschen an den Folgen der sanitären Versorgungskrise. Aus den Untersuchungen der BGR im Rahmen der TZ wird immer wieder deutlich, wie wichtig eine nachhaltige Sanitärversorgung für den Grundwasserschutz und damit auch für den langfristigen Schutz von Trinkwasservorkommen ist. Neben der erfolgreichen Verbreitung nachhaltiger Sanitärsysteme rücken in der TZ in den letzten Jahren daher vermehrt Aspekte des Angewandten Grundwasserschutzes in den Vordergrund. Die Implementierung dieser Aspekte ist hierbei Teil des größeren Prozesses des Integrierten Wasser-RessourcenManagements (IWRM), welcher alle Aspekte des Wasserkreislaufes beinhaltet. So gewinnt, neben Maßnahmen zur Beschreibung der Vulnerabilität (z.B. Ballungsräume in der Nähe hoch vulnerabler Karstgrundwasserleiter), die Nutzung gereinigter Abwässer in der Landwirtschaft zunehmende Bedeutung. Über Wege aus der sanitären Versorgungskrise diskutierten vom 14. bis 17. Oktober 2008 ca. 130 Experten beim internationalen Symposium „Coupling Sustainable Sanitation \& Groundwater Protection“, das von der BGR unter Mitwirkung des Bundesministeriums für wirtschaftliche Zusammenarbeit und Entwicklung (BMZ), des United Nations Environment Program (UNEP) und der World Health Organization (WHO) in Hannover organisiert wurde. Auf dem Symposium wurde deutlich, dass es eine Vielfalt technischer Lösungen für Sanitärsysteme gibt, die jedoch an den jeweiligen lokalen Kontext angepasst werden müssen, um nachhaltig sein zu können. Um die Nachhaltigkeitskriterien zu erfüllen, muss ein Sanitärsystem nicht nur ökonomisch, technisch und institutionell angepasst sein, sondern auch die natürlichen Ressourcen Grundwasser und Boden schützen. Im Hinblick auf die erfolgreiche Umsetzung des IWRM-Prozesses ergeben sich hieraus eine Fülle möglicher Kooperationsund Forschungsthemen.

Liebe Leserinnen und Leser, in meinen sicher persönlich gefärbten Ausführungen habe ich einige Themenfelder angerissen, die ich für die Hydrogeologie als wichtige Zukunftsfelder erachte. Die im Titel gestellte Frage über die zukünftige bessere interdisziplinäre Vernetzung der Hydrogeologie kann daher nur mit einem klaren Ja beantwortet werden. Zukunftsthemen wie die Adaption an den bereits stattfindenden Klimawandel, die Möglichkeiten des Integrierten Wasser-Ressourcen-Managements, die Maßnahmen zur Sicherung der Nahrungsmittelversorgung und ein verbesserter Grundwasserschutz sind sicher keine Themen, deren Besetzung wir anderen überlassen sollten. Lassen Sie uns in einen verstärkten Dialog treten, die Ergebnisse und Arbeitsthemen austauschen und mögliche Kooperationen ausloten. 